\title{
Additional information for "COVID-19 \\ fatality in Germany: Demographic \\ determinants of variation in case-fatality \\ rates across and within German federal \\ states during the first and second waves"
}

Saskia Morwinsky I morwinsky@demogr.mpg.de

Natalie Nitsche I nitsche@demogr.mpg.de

Enrique Acosta I acosta@demogr.mpg.de 


\section{Additional information for "COVID-19 fatality in Germany: Demographic determinants of variation in case-fatality rates across and within German federal states during the first and second waves"}

Saskia Morwinsky',2, 3, Natalie Nitsche², Enrique Acosta ${ }^{2}$

${ }^{1}$ University of Rostock, Rostock, Germany

${ }^{2}$ Max Planck Institute for Demographic Research, Rostock, Germany

${ }^{3}$ Interdisciplinary Centre on Population Dynamics, University of Southern Denmark, Odense, Denmark

This technical report provides details on methods and data sources for the study "COVID-19 fatality in Germany: Demographic determinants of variation in case-fatality rates across and within German federal states during the first and second waves", published in Demographic Research.

\section{German CFR from a European perspective}

Table S1 lists the CFR values across western European countries at the peak of the first pandemic wave and one year later. Data were retrieved from Our World in Data (Ritchie et al. 2020). The CFR estimates presented in Table S1 correspond to the ratio of confirmed COVID19 deaths to confirmed cases at a given time. Although there is a bias in these CFR estimates resulting from the lag between infection and death (i.e., individuals with confirmed infections who eventually died from COVID-19 but were alive at the date to which the CFR calculation refers, are included in the denominator but not in the numerator), the comparison across countries is valid because such a lag is equally implicit in all estimates.

Table S1. CFR values across western European countries at two time points.

\begin{tabular}{|l|c|c|}
\hline \multirow{2}{*}{ Country } & \multicolumn{2}{|c|}{ CFR } \\
\cline { 2 - 3 } & March 31, 2020 & March 31, 2021 \\
\hline Italy & $11.75 \%$ & $3.05 \%$ \\
\hline Spain & $8.82 \%$ & $2.30 \%$ \\
\hline Netherlands & $8.21 \%$ & $1.29 \%$ \\
\hline Sweden & $7.96 \%$ & $1.67 \%$ \\
\hline France & $6.75 \%$ & $2.04 \%$ \\
\hline United Kingdom & $6.33 \%$ & $2.91 \%$ \\
\hline Belgium & $5.52 \%$ & $2.61 \%$ \\
\hline Denmark & $2.96 \%$ & $1.05 \%$ \\
\hline Switzerland & $2.61 \%$ & $1.72 \%$ \\
\hline
\end{tabular}




\begin{tabular}{|l|l|l|}
\hline Ireland & $2.19 \%$ & $1.99 \%$ \\
\hline Portugal & $2.15 \%$ & $2.05 \%$ \\
\hline Austria & $1.26 \%$ & $1.71 \%$ \\
\hline Finland & $1.20 \%$ & $1.09 \%$ \\
\hline Germany & $\mathbf{1 . 0 8} \%$ & $\mathbf{2 . 6 9 \%}$ \\
\hline Norway & $0.84 \%$ & $0.70 \%$ \\
\hline
\end{tabular}

\section{Definition of confirmed COVID-19 cases, recoveries, and deaths in Germany}

According to the Robert Koch Institute (RKI), confirmed COVID-19 cases are defined as those cases in which a PCR laboratory test returns a positive result (RKI 2020). Confirmed COVID19 deaths are defined as deaths in which there is evidence of laboratory-confirmed SARSCoV-2 (either pre- or post-mortem) and a medical diagnosis of death in relation to this infection. Deaths in which it cannot be conclusively determined whether the infected person died as a consequence of the disease are also recorded as confirmed COVID-19 deaths (RKI 2021a). Regarding the definition of recoveryfrom the disease, it is assumed that for mild cases (i.e., without further complications), the illness lasts for 14 days; whereas for cases with symptoms indicating severe complications, such as pneumonia, the duration of the disease is extended to 28 days. After those periods, if there is no notification of COVID-19-related death, the individuals in these cases are considered to have recovered (RKI 2021b). 
State-specific age distribution of the population, and confirmed cases and deaths over time between March 2020 and March 2021

Figure S1. Age distribution of the population, and confirmed cases and deaths over time in each German state between March 2020 and March 2021.

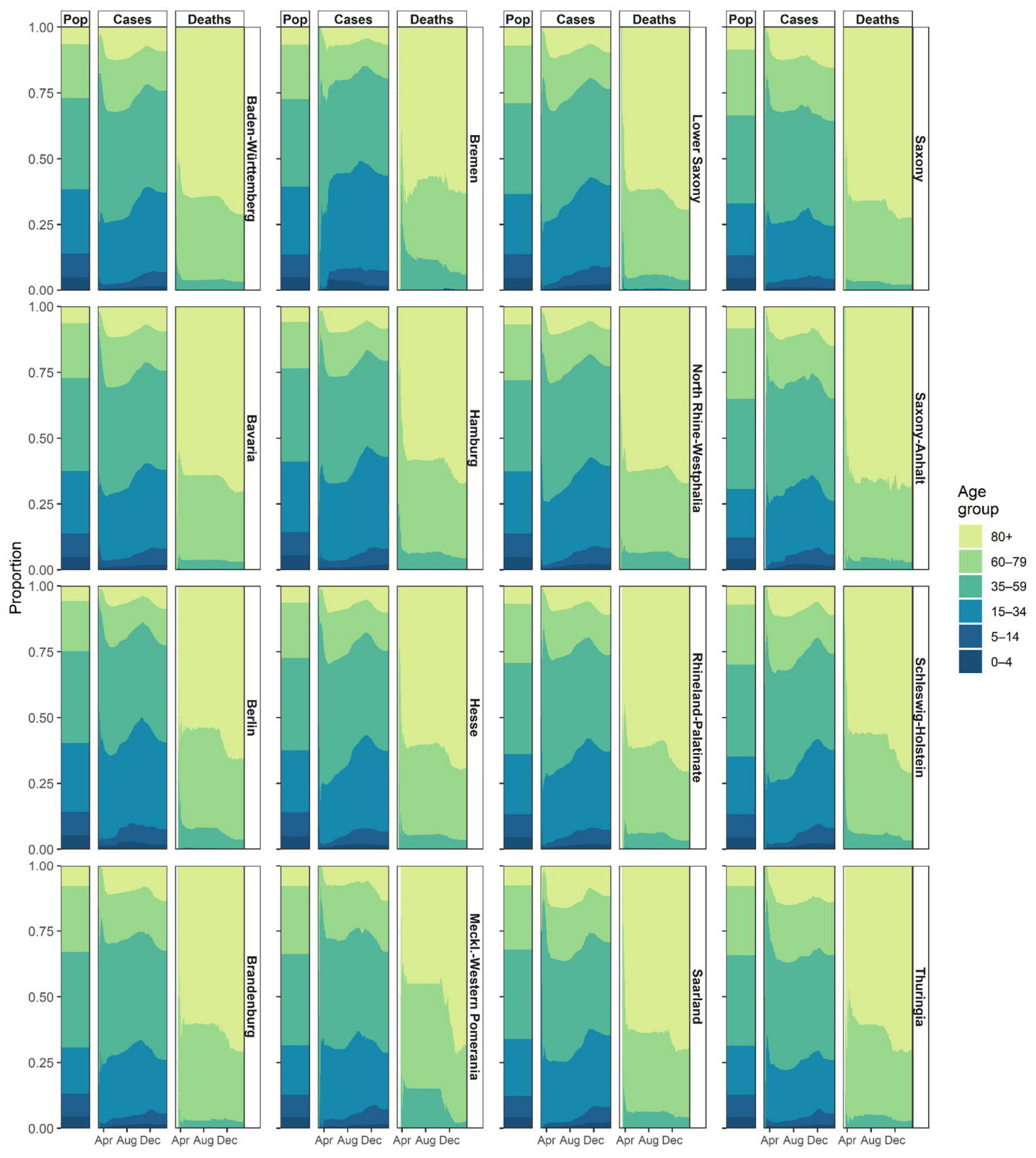




\section{Sensitivity analyses of the decomposition of CFR changes within each German state over time}

In this section, we evaluate the sensitivity of the decomposition of CFR changes over time estimates to a) changes in the population age structure over time, and b) and the selection of a unique time window for all states.

\section{a) Assumption of a constant population age composition over time}

To evaluate the sensitivity of the results to the assumption of a constant population age structure during the pandemic, we incorporate two sources of population variation during the observation period. First, we incorporate population changes that were expected to gradually occur according to population projections. Second, we incorporate population changes that resulted from mortality changes that were direct and indirect consequences of the COVID-19 pandemic.

Expected daily changes in the population by age in each state were estimated by interpolating annual population estimates on December 31 of each year between 2018 and 2020 (Destatis 2021), and extrapolating the values until May 2021. The interpolation and extrapolation estimates were obtained using cubic splines. Excess mortality estimates at the national level were obtained from Acosta (2021), who reported a total excess of 50,000 deaths between March 2020 and April 2021. The excess deaths were distributed among states based on the share of confirmed deaths in each age interval, and were distributed over time according to the temporal dynamics of confirmed COVID-19 mortality.

Figure S2 presents the Horiuchi decomposition of CFR changes over time within each German state during the periods of CFR decreases (left column) and increases (right column). The estimates in the first-row panel represent the results of our original analysis (as depicted in Figure 5 in the main manuscript), which were obtained with a population that is assumed to be constant over time, while the estimates in the second-row panel are based on a decomposition analysis with a population that changes over time due to both expected and COVID-19-related variations. In other words, this time-varying population incorporates both sources of variation simultaneously: the expected population change based on prior changes and pandemic-related excess mortality. These results suggest that the contribution of population change to CFR changes over time (depicted by purple bars in the second-row panel) is negligible, ranging from one to five percent, with an average of two percent.

\section{b) Differences in the observation time window across German states}

In order to test how sensitive the decomposition estimates of CFR changes over time are to the differences in the definition of the observation time spans across states, we performed the same decomposition using common time windows for all states when decomposing the decreases and increases in CFRs within states. For defining the common time span for all states, instead of selecting the dates at which each state reached the maximum and minimum CFR values, we selected the middle dates at which these levels were attained in all states. The first period covers April 20 to November 8, 2020; and the second period covers November 8, 2020, to February 4, 2021. Again, the first-row panel in Figure S2 depicts the original 
decomposition estimates when using state-specific time windows, whereas the third-row panel shows the estimates when using common time windows for all states. Small changes can be seen as resulting from the imposition of a common observation period. However, the estimates from the two decompositions are consistent, and suggest that the selected state-specific time windows are not determinants of the observed decomposition estimates of CFR changes. 
Figure S2. Sensitivity analyses of the decomposition of CFR changes within each German state over time.

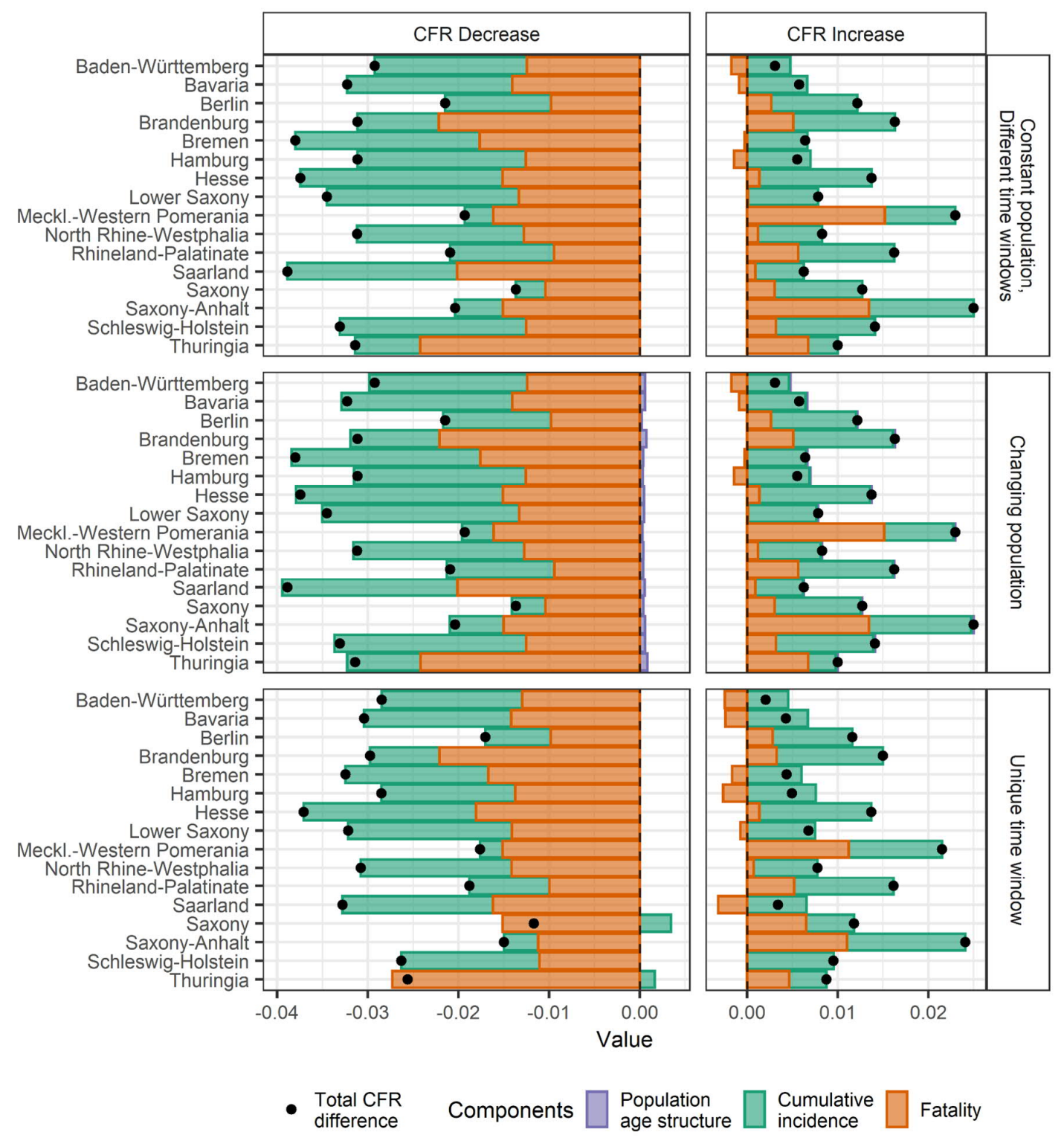

Note: The first-row panel depicts the original estimates under the assumption of a constant population age composition over time and state-specific observation time windows. The second-row panel includes changes in the age structure of the population. The third-row panel shows the estimates when common time windows are defined in all states. The dots indicate the total CFR difference. The bars indicate the contributions to the total CFR difference of population age structure differences (in purple), the age structure of confirmed infection rates differences (in green), and the differences in the age-specific fatality rates (in orange). The negative and positive values indicate, respectively, decreasing and increasing CFRs. The whole period under analysis covers the time period between April 13, 2020, and March 8, 2021. More details on the dates covered in each state are listed in Figure 5 in the main text.

Source: COVerAGE-DB (own analyses and illustration). 


\section{Influence of testing COVID-19 coverage on CFR levels}

Because the identification of confirmed cases occurs through testing, it is possible that the CFR changes were mainly driven by changes in the testing intensity. In other words, a higher level of testing coverage would lead to a higher level of infection detection among less vulnerable segments of the population, which would, in turn, reduce the CFR levels.

To explore how sensitive the CFR levels are to the testing intensity across German states, the scatterplots in Figure S3 correlate the CFR levels and testing coverage in all 16 German states on three state-specific dates: 1) when the CFR attained the maximum level during the first wave, 2) when it reached the minimum level between waves, and 3) when it attained a maximum level again during the second wave.

The lack of a clear and strong correspondence in these plots between the two measures suggests that differences in overall testing intensity are not the main driver of differences in CFR levels. We estimated a Spearman coefficient in each period to evaluate the correlation between testing intensity and CFR levels (indicated in the upper-right corner of each panel in Figure S3). These coefficients are consistent with the absence of clear correlations, which were already visually noted in the scatterplots. There is a small negative correlation ranging between -0.23 and -0.24 during the two first periods under analysis, and a virtual absence of correlation during the third period, with a Spearman coefficient of only -0.035 .

Figure S3. Testing coverage and CFR values by German states in the periods in which each CFR in each state attained the maximum (Periods 1 and 3) and minimum (Period 2) levels.

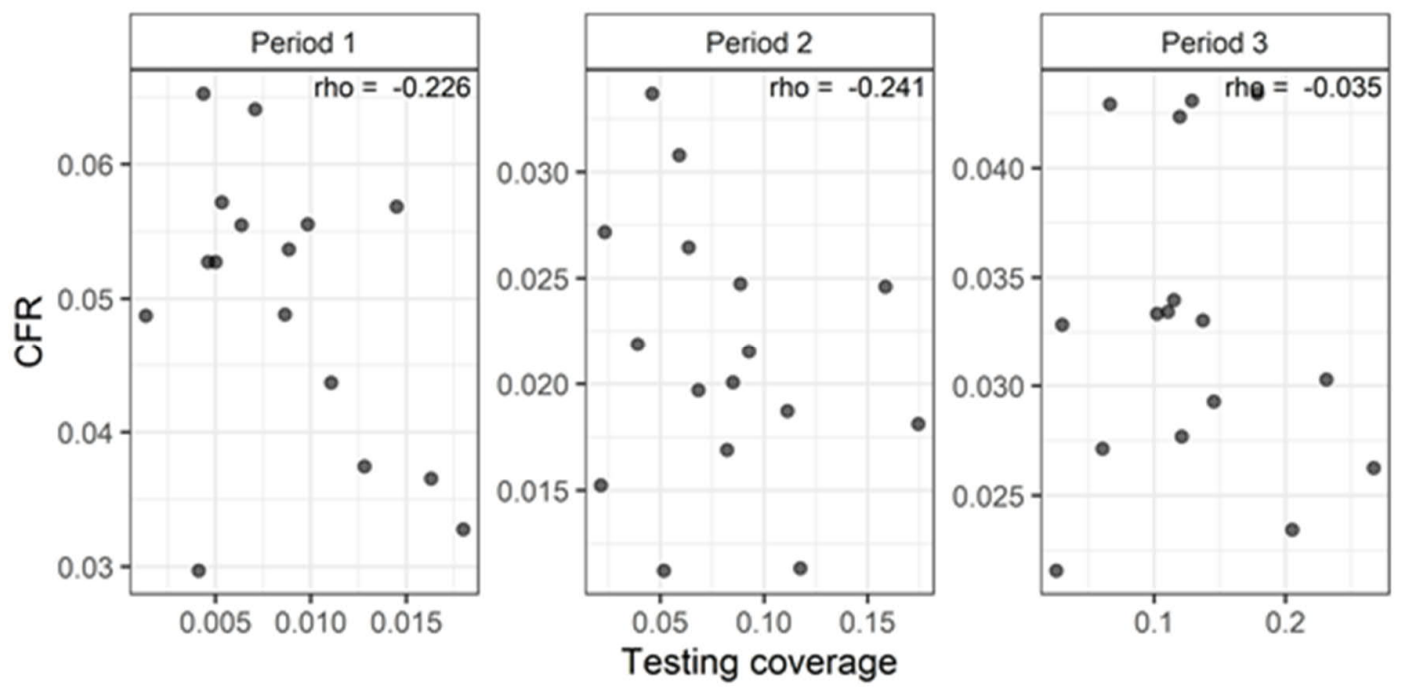

Note: The three periods correspond to the date on which each state attained the maximum CFR value in the first wave (Period 1, between April 19 and May 31, 2020), the minimum CFR value between the first and second waves (Period 2, between October 18 and November 29, 2020), and the maximum CFR value during the second wave (Period 3, between January 24 and March 7, 2021).

Figure S4 plots the daily cumulative CFR value (in the $y$-axis) according to the testing coverage level (in the x-axis) in each state. Two observations are noteworthy: 1) for the same level of testing coverage, there is wide variation in CFR levels across states, and 2) the increase in testing coverage does not necessarily translate into CFR reductions. 
Figure S4. Daily CFR values and testing coverage by German state between May 2020 and April 2021

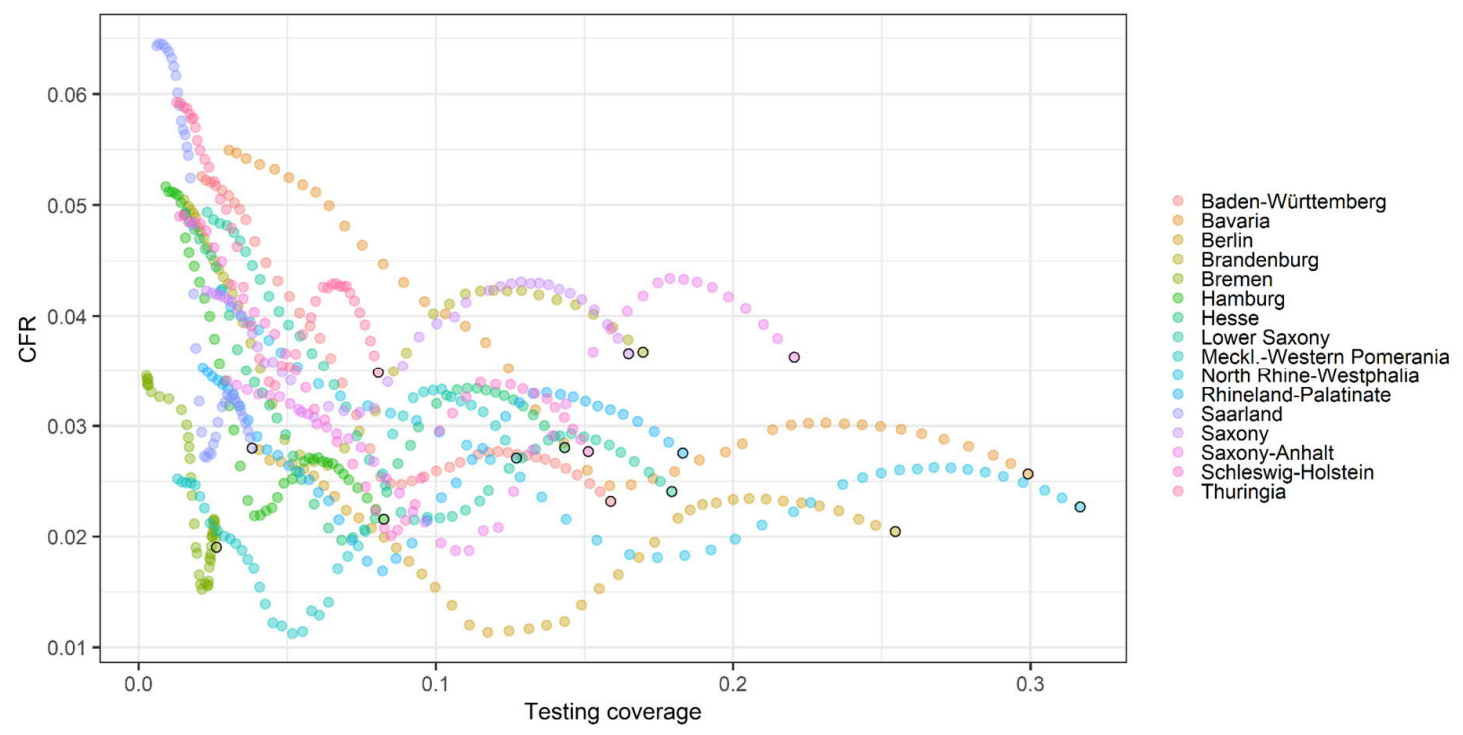

Note: The black border circles indicate the last observation in each state.

An analysis of the changes in the age composition of testing would allow us to better understand the influence of variations in testing regimes on CFR differences across states and over time. Unfortunately, the lack of data on age-specific testing by German states makes it impossible to analyze this relationship.

\section{References}

Acosta, E. (2021). Excess Mortality by Sex and 5-year Age Groups [electronic resource]. OSF. https://github.com/kikeacosta/excess_mortality/tree/bad697002f759a642efcfdebddd0 $498 c 4$ e9bdf2a.

Destatis (2021). Statistisches Bundesamt Deutschland - GENESIS-Online [electronic resource]. https://wwwgenesis.destatis.de/genesis/online?sequenz=tabelleAufbau\&selectionname=12421 0004\#astructure.

Ritchie, H., Ortiz-Ospina, E., Beltekian, D., Mathieu, E., Hasell, J., Macdonald, B., Giattino, C., Appel, C., Rodés-Guirao, L., and Roser, M. (2020). Coronavirus Pandemic (COVID-19). Our World in Data. https://ourworldindata.org/coronavirus.

RKI (2020). Coronavirus-Krankheit-2019 (COVID-19) (SARS-CoV-2). Robert Koch-Institut: Robert Koch-Institut. https://www.rki.de/DE/Content/InfAZ/N/Neuartiges_Coronavirus/Falldefinition.pdf? blob=publicationFile.

RKI (2021a). RKI - Coronavirus SARS-CoV-2 - Fallzahlen und Meldungen (Stand: 23.7.2021) [electronic resource]. https://www.rki.de/SharedDocs/FAQ/NCOV2019/FAQ_Liste_Fallzahlen_Meldungen. html.

RKI (2021b). Tabelle mit den aktuellen Covid-19 Infektionen pro Tag (Zeitreihe). [electronic resource]. https://www.arcgis.com/home/item.html?id=dd4580c810204019a7b8eb3e0b329dd6\# overview. 